\title{
Resorption of roots of different dimension induced by different types of forces
}

\section{Osmar Aparecido CUOGHI(a) \\ Carlos Alberto AIELLO(b) \\ Alberto CONSOLARO(c) \\ Pedro Marcelo TONDELLI(d) \\ Marcos Rogério de MENDONÇA ${ }^{(a)}$}

\footnotetext{
(a) Department of Pediatric and Community Dentistry, Faculdade de Odontologia de Araçatuba, Universidade Estadual Paulista UNESP, Araçatuba, SP, Brazil.

(b)Department of Orthodontics, Hospital de Reabilitação de Anomalias Craniofaciais HRAC, Universidade de São Paulo - USP, Bauru, SP, Brazil.

(c)Department of Stomatology, Faculdade de Odontologia de Bauru, Universidade de São Paulo - USP, Bauru, SP, Brazil.

(d) Department of Oral Medicine and Pediatric Dentistry, Centro de Ciências da Saúde, Universidade Estadual de Londrina - UEL, Londrina, PR, Brazil.
}

Declaration of Interests: The authors certify that they have no commercial or associative interest that represents a conflict of interest in connection with the manuscript.

Corresponding Author:

Carlos Alberto Aiello

E-mail: aiello@usp.br

DOI: 10.1590/1807-3107BOR-2014.vol28.0013 Epub: Jun 02, 2014

Submitted: May 08, 2013

Accepted for publication: Jan 21, 2014

Last revision: Mar 28, 2014

\begin{abstract}
Root resorption is a variable to be considered in induced tooth movement (ITM). It is related to root morphology and alveolar bone crest, and also to the types of forces exerted by mechanotherapy. This histometric study evaluated the predominance of root resorption among roots of different dimensions, following ITM with different types of forces and at different time intervals. The study was conducted on 54 rats divided into three groups, according to the type of force: continuous (CF), continuous interrupted (CIF) and intermittent (IF), at periods of 5, 7 and 9 days. The percentage of resorption between mesiobuccal roots of larger dimension and intermediate roots of smaller dimension was assessed. The evaluations were performed on the AxioVision software, and the non-parametric analysis of variance for repeated measures in independent groups was further applied, consisting of a scheme of two factors, and complemented by the Dunn test at a significance level of $5 \%$. The intermediate roots presented a higher percentage of resorption, which was gradual at the periods evaluated for the three types of forces, but mainly for CF. Comparing the intermediate roots with the mesiobuccal roots, there was a statistically significant difference $(p<0.05)$ in the CF group at day 7 and day 9 , and in the FI group, at day 9. The intragroup analysis evidenced a statistically significant difference $(p<0.05)$ between the $5^{\text {th }}$ and the $9^{\text {th }}$ day for the intermediate root in the CF group. The intergroup analysis did not reveal any statistically significant difference $(p>0.05)$ in individually analyzed roots.
\end{abstract}

Keywords: Root Resorption; Tooth Movement; Rats.

\section{Introduction}

Orthodontic mechanics involves continuous, continuous interrupted or interrupted and intermittent forces. These forces cause compression and tensile stresses on the periodontal ligament and alveolar bone, inducing morphological and microscopic reactions controlled by chemical mediators, promoting tooth displacement, ${ }^{1,2,3}$ which is called orthodontic tooth movement or induced tooth movement (ITM).

The mechanism and biological reaction of ITM depend on the magnitude, duration and type of force delivered by stainless stee ${ }^{4,5}$ wires and springs, by superelastics with shape memory, like nickel-titanium alloys (NiTi), ${ }^{5,6,7,8}$ by $\beta$-titanium or titanium-molybdenum (TMA), ${ }^{9}$ or by intraand intermaxillary elastics. These forces can induce variable degrees of root resorption ${ }^{4,5,8,10}$ in different root morphologies. .0,11,12 $^{2}$ 
Root morphology may influence root resorption; i.e., teeth with short roots, a triangular shape, pipetteshaped apical thirds or dilaceration pose higher risk of resorption. ${ }^{3,13}$ Dental traumas, ${ }^{14}$ as well as the alveolar crest morphology, ${ }_{1}^{11}$ should also be considered risk factors in root resorption.

The extent of root resorption in ITM can be evaluated by different methods and imaging techniques.,12 The most indicated methods are periapical radiographs and cone-beam computed tomography (CBCT), which allow accurate diagnosis in three-dimensional images. ${ }^{11,15,16}$ Panoramic radiographs or cephalograms have limitations regarding methodology and accuracy, and thus should be analyzed carefully when assessing their results.

Both the tissue phenomena and the root resorption related to ITM can be investigated in experimental models in rats, by induced movement of the maxillary first molar, ${ }^{17,18,19,20,21}$ and be extrapolated to humans.

This study evaluated the predominance of root resorption among roots of different dimensions during ITM of the maxillary right first molar in rats, using different types of forces and time periods.

\section{Methodology}

The sample included 54 male young adult Wistar rats (Rattus norvegicus albinus). This study was approved by the Institutional Review Board on Animal Studies (Faculdade de Odontologia de Araçatuba, UNESP), under Protocol no. 004938-2009.

The sample was divided into three groups according to the type of force: continuous (CF), continuous interrupted (CIF) and intermittent (IF). Each group was divided into three subgroups with six animals each, to evaluate changes occurring at periods of 5, 7 and 9 days.

In the CF group (Figure 1A), the springs were maintained without force interruption until the $5^{\text {th }}$, the $7^{\text {th }}$ and the $9^{\text {th }}$ days. In the CIF group (Figure 1B), the springs were deactivated after three days of continuous force, and maintained in this passive position until the $5^{\text {th }}$ day, when they were reactivated and maintained in this state until the $7^{\text {th }}$ day, then deactivated again until the $9^{\text {th }}$ day. The same procedure was carried out in the IF group (Figure 1C), and in the CIF group, except in regard to the springs, which were removed and replaced.

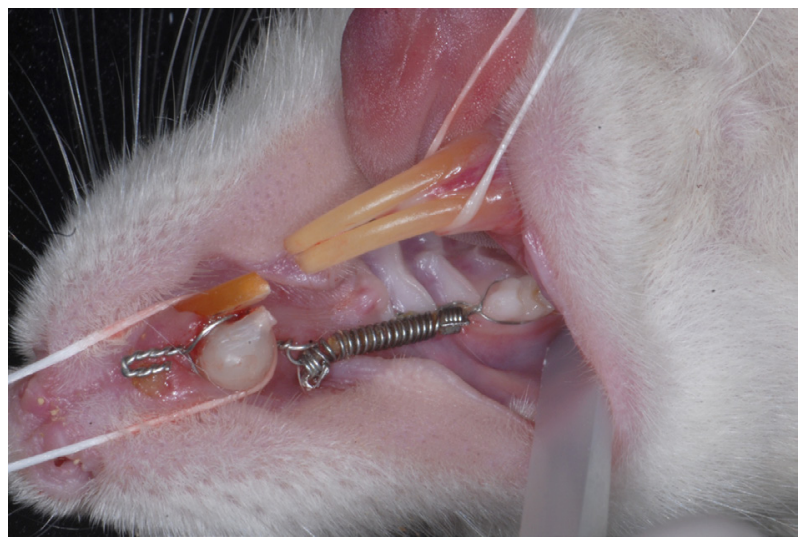

Figure 1A. Continuous force: activated spring

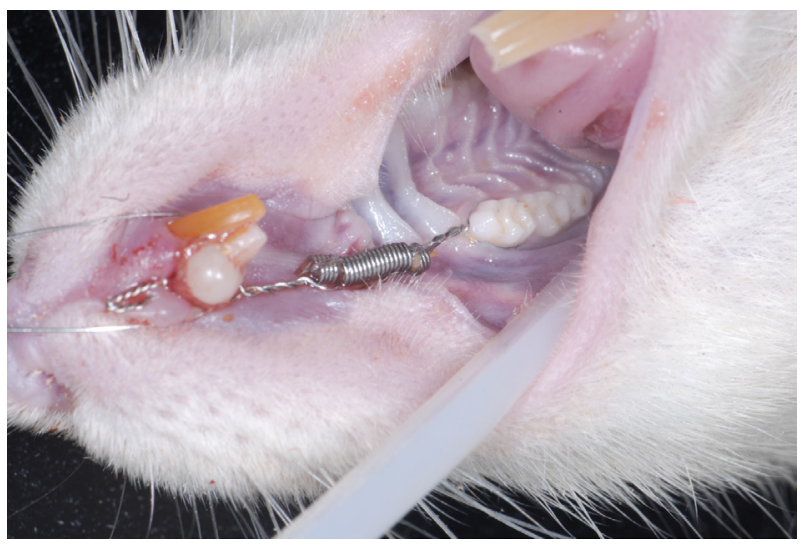

Figure 1B. Continuous interrupted force: non-activated spring in a passive position

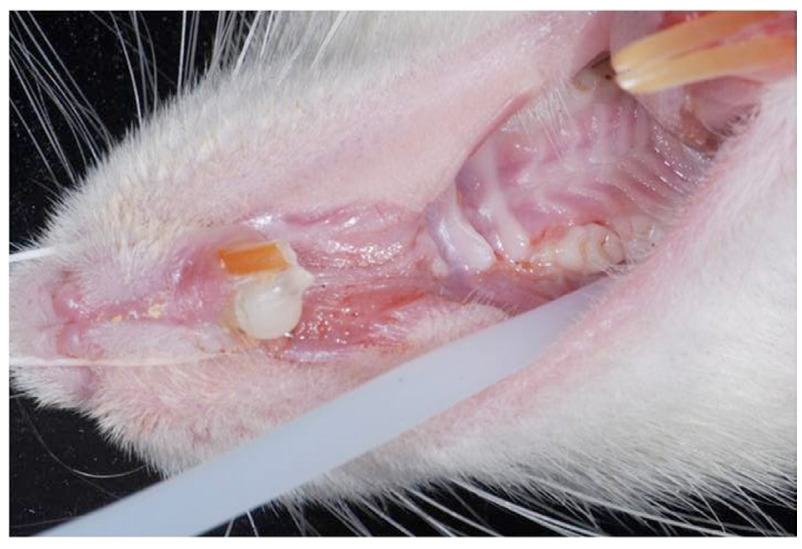

Figure 1C. Intermittent force: removed spring

The experimental procedure was divided into two stages. In the first, the murines were 62 days old, and the procedure comprised of inducing dentoal- 
veolar ankylosis of maxillary right incisors (Figure 2) to promote absolute anchorage for movement of the maxillary right first molar, without influencing the type of force applied during movement, especially CIF. This stage was performed according to a predetermined protocol: ${ }^{22}$ the anesthetic (Dopalen - ketamine hydrochloride) and the muscle relaxant (Anasedan - xylazine hydrochloride) - Vetbrands, Paulínia, Brazil - were mixed and applied by intramuscular injection, followed by luxation and extraction, removal of periradicular tissues and dental papilla, root canal obturation with a mixture of calcium hydroxide and propylene glycol, and root apex sealing with a mixture of grey MTA (Angelus, Londrina, Brazil) and distilled water, and replantation.

A period of two weeks was allowed for formation of ankylosis before the second stage was initiated. This consisted of ITM of the maxillary right first molar when the murines were 76 days old, with a mean weight of $274.75 \mathrm{~g}$, using three different types of forces. The procedure was performed with a 3-mm nickel-titanium closed coil spring Sentalloy ${ }^{\circledR}$ (GAC) (COIL SPRINGS 10-000-25 - GAC International Inc., New York, USA), to deliver a continuous force of $50 \mathrm{cN}$, adapted to the cervical region of the maxillary right first molar with a $0.20-\mathrm{mm}$ diameter stainless steel wire, and to the maxillary right incisor with a $0.25-\mathrm{mm}$ diameter stainless steel wire. This method followed the principles of Heller and Nanda ${ }^{17}$ with innovative changes.

The animals were euthanized by injection of an excessive anesthetic dose on completion of each experiment, and histotechnical processing was then performed.

The specimens embedded in paraffin blocks were transversely and serially sectioned at $6 \mu \mathrm{m}$. All roots of the maxillary right first molar were separated at the cervical third, immediately below the alveolar bone crest level, where homogeneous structures of alveolar bone tissue and periodontal ligament were observed extending to the incisor roots, and were stained with hematoxylin-eosin (Figure 2).

\section{Histometric analysis}

The sections (Figure 2) were examined under a ZEISS Axiophot (Carl Zeiss, Göttingen, Germany) microscope and images of the mesiobuccal and intermediate roots at $25 \mathrm{x}$ magnification were captured by a digital camera connected to the microscope.

After acquiring the images, the mesiobuccal and intermediate roots (Figure 3) were visualized using

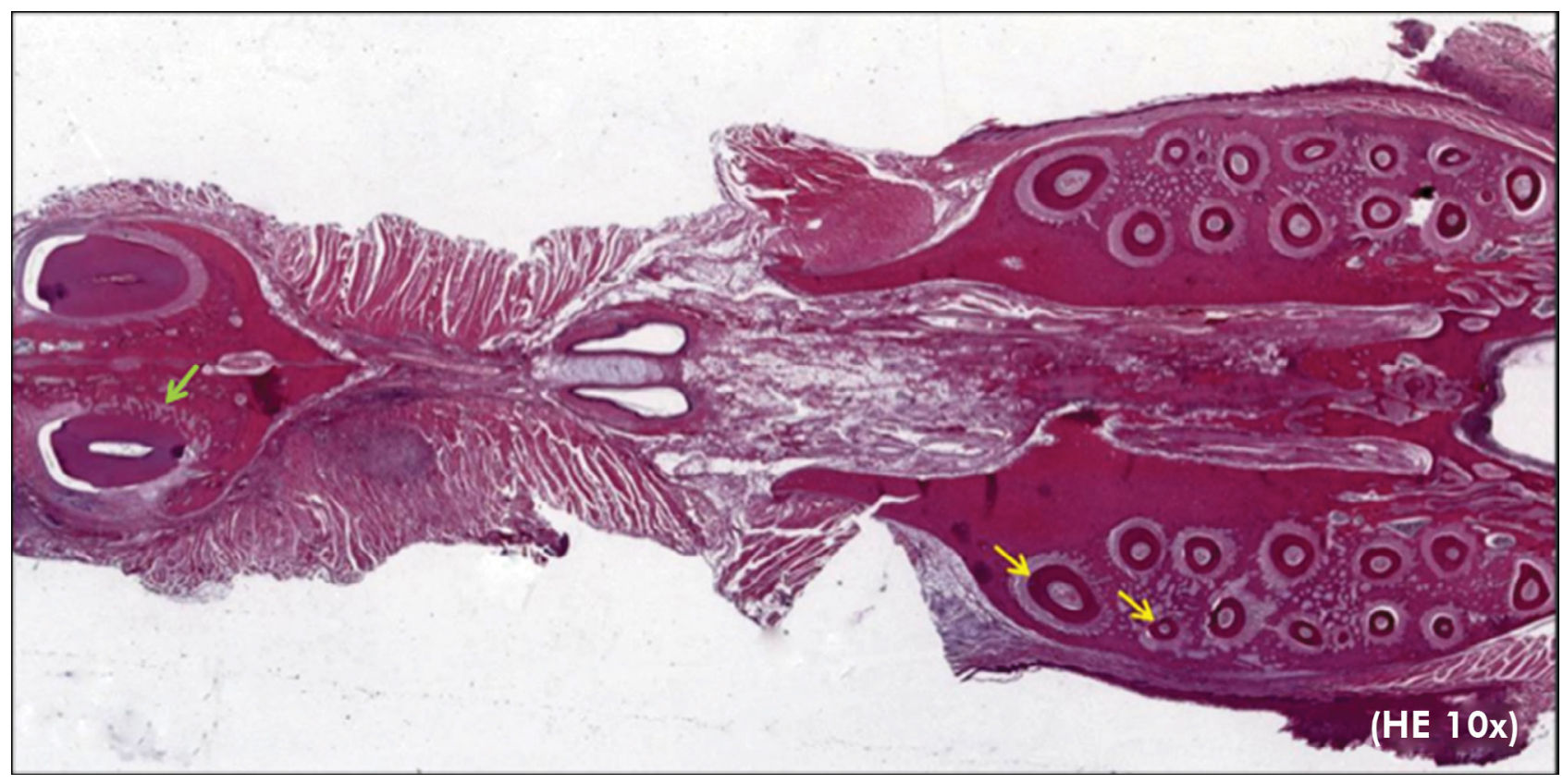

Figure 2. Section evidencing the mesiobuccal and intermediate roots (yellow arrows) and maxillary right incisor with dentoalveolar ankylosis (green arrow). HE 10x 


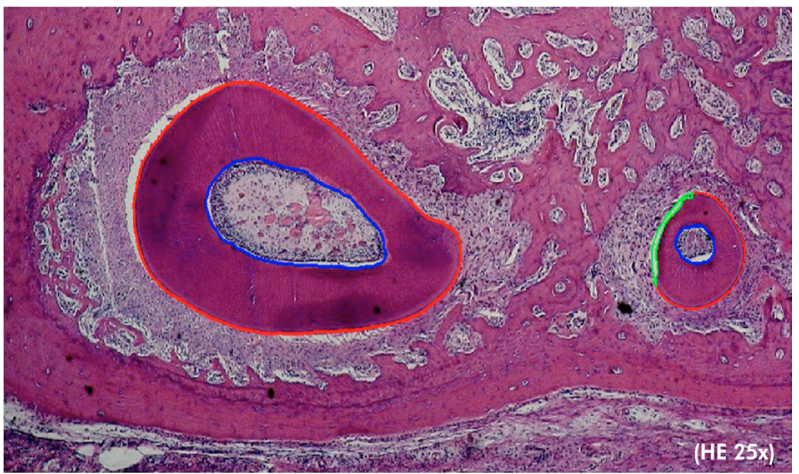

Figure 3. Contour of the greatest root perimeter and most external limit of cementum (in red); resorption area (green) and internal dentin contour (blue) surrounding the pulp externally. HE 25x

a microcomputer, demarcated and measured with Imaging Systems - Software Release 4.8.2 - AxioVision (Copyright 1999-2010 by Carl Zeiss, Göttingen, Germany) software to quantify the percentage of resorption in each period of ITM. The greater root perimeter was then contoured at the most external limit of cementum, in red. The resorbed root area was contoured internally in green, and the original root contour was simulated externally. Then, another contour was made in blue, delimiting the smaller perimeter at the most internal dentin limit, surrounding the pulp externally (Figure 3).

This procedure allowed calculation of the total area of root dentin and cementum, as well as the areas of resorption, expressed in square micrometers. Accordingly, the percentage of root resorption of the two roots was calculated for each type of force at the different time intervals.

\section{Evaluation of measurement error}

For the purpose of method error analysis, $36 \mathrm{sec}-$ tions were randomly selected and the two roots were once again measured after a one-week interval.

The intraexaminer systematic error was calculated by the Wilcoxon Signed Rank Test. The casual error was determined using the error calculation proposed by Dahlberg. ${ }^{23}$

$$
\text { error }=\sqrt{\frac{\sum d^{2}}{2 n}}
$$

where $\mathrm{d}=$ difference between first and second measurements

$\mathrm{n}$ = number of repetitions

Table 1 shows the method error. The probability values correspond to the systematic error, whereas the values obtained by the Dahlberg formula represent the casual error. No systematic or casual errors were observed, and results considered within acceptable parameters were obtained, thus indicating the reliability of the present conclusions.

Table 1. Median, minimum and maximum values, expressed in percentage of measurements of mesiobuccal (m) and intermediate (i) roots.

\begin{tabular}{|c|c|c|c|c|}
\hline \multirow[t]{2}{*}{ Root } & \multicolumn{2}{|c|}{ Measurement } & & \multirow{2}{*}{$\begin{array}{c}\text { Dahlberg } \\
\text { error }\end{array}$} \\
\hline & $1^{\text {st }}$ & $2^{\text {nd }}$ & & \\
\hline (m) root & $0.00(0.00 ; 1.01)$ & $0.00(0.00 ; 1.07)$ & $\begin{array}{c}p=0.63 \\
\text { (ns) }\end{array}$ & $0.02 \%$ \\
\hline (i) root & $0.74(0.00 ; 4.71)$ & $0.58(0.00 ; 3.66)$ & $\begin{array}{c}p=0.54 \\
\text { (ns) }\end{array}$ & $0.29 \%$ \\
\hline
\end{tabular}

\section{Statistical analysis}

Comparison of the percentage of root resorption, according to the type of mechanical force, study period and type of root, was made initially applying the Kolmogorov-Smirnov test to analyze the adherence of data in the normal distribution of probabilities. Since the statistical test revealed abnormal distribution, nonparametric analysis of variance for repeated measures in independent groups was further applied, consisting of the two-factor scheme (type of force and study period). The analysis was complemented by the Dunn multiple comparisons test, at a significance level of $5 \%{ }^{24}$

\section{Results}

In Table 2, the intragroup analysis showed that the intermediate root presented a higher percentage value of resorption for the three types of forces, as compared with the mesiobuccal root, except for day 5 in the intermittent force group. The statistical analysis, represented by Greek letters, indicated a significant difference $(p<0.05)$ at day 7 and day 9 in the continuous force group, and at day 9 in the intermittent force group. 
The comparison among the three study periods for each type of force and the root analyzed, performed for the intragroup analysis and represented by lowercase Arabic letters, did not reveal any statistically significant result $(p>0.05)$ for the mesiobuccal root. In regard to the intermediate root, a statistically significant difference $(p<0.05)$ was observed for the continuous force (CF) group between the $5^{\text {th }}$ and the $9^{\text {th }}$ days. Even though no statistically significant difference was observed for this root between the $7^{\text {th }}$ and the $9^{\text {th }}$ days, from a practical standpoint, there was an important increase in the numerical values of the medians ( 0.69 and 1.76 ), respectively. No statistically significant results were observed for the other two forces (CIF and IF) $(p>0.05)$.

The comparison among the same three study periods for the three types of forces, performed for the intergroup analysis and represented by uppercase Arabic letters, did not reveal any statistically significant difference $(p>0.05)$ in individually analyzed roots. The highest numerical values of the medians were observed in the intermediate root for the continuous force group.

Table 2. Median, minimum and maximum values of root resorption expressed in percentage according to type of force, study period and evaluated root.

\begin{tabular}{lllc}
\hline \multirow{2}{*}{ Force } & Period & \multicolumn{2}{c}{ Root } \\
\cline { 3 - 4 } CF & 5 days & $0.00(0.00 ; 0.44)$ a a A & $0.44(0.00 ; 2.75)$ a a A \\
& 7 days & $0.10(0.00 ; 0.35)$ a a A & $0.69(0.16 ; 3.38) \beta$ ab A \\
& 9 days & $0.00(0.00 ; 0.55)$ a a A & $1.76(1.06 ; 7.81) \beta$ b A \\
\hline CIF $\quad 5$ days & $0.00(0.00 ; 0.38)$ a a A & $0.06(0.00 ; 1.20)$ a a A \\
& 7 days & $0.00(0.00 ; 0.74)$ a a A & $0.36(0.00 ; 5.08)$ a a A \\
& 9 days & $0.02(0.00 ; 0.63)$ a a A & $0.83(0.00 ; 7.56)$ a a A \\
\hline IF $\quad 5$ days & $0.00(0.00 ; 0.44)$ a a A & $0.00(0.00 ; 2.73)$ a a A \\
& 7 days & $0.06(0.00 ; 0.46)$ a a A & $0.19(0.00 ; 2.80)$ a a A \\
& 9 days & $0.00(0.00 ; 1.01)$ a a A & $0.41(0.00 ; 2.30) \beta$ a A \\
\hline
\end{tabular}

The Greek letter enabled interpretation of the intragroup analysis, which compared the mesiobuccal and intermediate roots with each other, according to the type of force and to the different study periods. Two medians followed by the same Greek letter do not present any statistical difference $(p>0.05)$.

The lowercase Arabic letter enabled interpretation of the intragroup analysis, which compared the different study periods according to each type of force and root analyzed. Two medians followed by at least one same lowercase Arabic letter do not present any statistical difference $(p>0.05)$. The uppercase Arabic letter enabled interpretation of the intergroup analysis, which compared the same study periods according to the different forces for each root analyzed. Two medians followed by the same uppercase Arabic letter do not present any statistical difference $(p>0.05)$.

\section{Discussion}

The modifications to the principles of Heller and Nanda ${ }^{17}$ in this study allowed absolute anchorage, prevented continuous eruption of incisors and maintained constant force levels during the ITM of the maxillary right first molar. Selection of the transverse section at the cervical region of the roots was based on concomitant visualization of the roots of interest, observation of the entire root perimeter and quantity and quality of acellular cementum, which is similar to humans in rats.

Induced tooth movement occurs by application of mechanical forces, ${ }^{1,2,3,4,13,18}$ which create areas of tensile stress and compression. Depending on the type of force, magnitude and duration, cementum resistance to resorption may be disrupted by local clast activity, leading to dentin resorption. ${ }^{1}$

During the application of orthodontic forces, triangular bone crests tend to undergo greater deflection, as compared with rectangular- or rhomboidal-shaped crests. Since these crests absorb part of this force, they tend to distribute it with lower risk both of lesion to the cementoblast layer and of root resorption. This may justify the greater root resorption percentage in intermediate roots (Table 2), in which the interradicular septa present a rhomboidal or rectangular shape, as compared with mesiobuccal roots, which present a triangular shape on the mesial surface. In addition, because mesiobuccal roots are larger, as compared with intermediate roots, there is better force dissipation, with fewer undesirable effects.

Another aspect refers to the type of movement generated when the first molars are moved in an anterior or a mesial direction. The trapezoidal shape of these molars causes a tipping movement, with extrusion in the distal region, thus increasing susceptibility to trauma during chewing, as well as greater compression of the mesial periodontal ligament in intermediate and distal roots.

A recent study ${ }^{25}$ revealed that the periodontal ligament thickness in rats is proportional to the root dimensions, i.e., smaller roots present a smaller thickness of periodontal ligament, as compared with larger roots. This is probably the most important factor explaining the higher susceptibility of interme- 
diate roots to resorption, as compared with mesiobuccal roots, considering the same force magnitude of $50 \mathrm{cN}$ applied to both roots.

Although some studies show better results in relation to the extent of tooth movement by using NiTi or TMA wires, ${ }^{5,8,9}$ a continuous interrupted force may be considered the most favorable option, especially in relation to lower predominance of root resorption. . $^{1,59}$

Comparing intermittent and continuous forces, Ballard et al. ${ }^{9}$ reached the same conclusion. That is to say, a continuous force $(\mathrm{CF})$ presented better results in relation to the extent of tooth movement, but induced greater root resorption. This study revealed a tendency for higher percentage values of resorption when CF was used (Table 2); CF presented statistically significant differences between the $5^{\text {th }}$ and the $9^{\text {th }}$ days for intermediate roots.

In relation to continuous interrupted and intermittent forces, in this study, a gradual increase was observed in the numerical percentages of resorption in intermediate roots, when compared to the $5^{\text {th }}$, the $7^{\text {th }}$ and the $9^{\text {th }}$ days. Despite the lack of any statistically significant difference, the importance of this increase should not be ruled out.

Although the results for the intermediate roots (Table 2) in the intergroup analysis indicated no statistically significant difference, they revealed that continuous force induced the highest percentage of resorption, as compared with the other types of forces, in all the periods.

Thus, notwithstanding the lack of any statistically significant difference, continuous force tends to be unfavorable as regards risk of root resorption, despite the more favorable results in relation to the extent of orthodontic movement. ${ }^{5,8,9}$

\section{References}

1. Krishnan V, Davidovitch Z. Cellular, molecular, and tissuelevel reactions to orthodontic force. Am J Orthod Dentofacial Orthop. 2006 Apr;129(4):469.e1-32.

2. Cattaneo PM, Dalstra M, Melsen B. Strains in periodontal ligament and alveolar bone associated with orthodontic tooth movement analyzed by finite element. Orthod Craniofac Res. 2009 May;12(2):120-8.
Another aspect is that the median values in the intermittent force group were the smallest, as compared with the other forces. Despite the lower aggressiveness of this force in regard to root resorption, the results regarding the extent of orthodontic movement for this type of force are less favorable., ${ }^{91}$

This study demonstrated that continuous interrupted force promoted intermediate values of root resorption, despite the smaller extent of movement, as compared with only continuous force. ${ }^{5}$ Therefore, based on the present results, as applied to rat teeth, continuous interrupted force was a good method that could be applied to induce tooth movement in order to lessen the risk of root resorption.

\section{Conclusions}

1. The intermediate root presented a higher percentage of root resorption, as compared with the mesiobuccal root, mainly after application of continuous force.

2. The intermediate root presented a gradual increase in root resorption in the three study periods for the different types of forces.

3. The fact that continuous interrupted force exhibited intermediate values among the different types of forces did not rule out the possibility of root resorption.

\section{Acknowledgements}

We wish to thank Fundação para o Desenvolvimento da Unesp (Fundunesp) for the financial support given to this study (Process no. 01214/08).

3. Brezniak N, Wasserstein A. Orthodontically induced inflammatory root resorption. Part I: the basic science aspects. Angle Orthod. 2002 Apr;72(2):175-9.

4. Reitan K. Initial tissue behavior during apical root resorption. Angle Orthod. 1974 Jan;44(1):68-82.

5. Weiland F. Constant versus dissipating forces in orthodontics: the effect on initial tooth movement and root resorption. Eur J Orthod. 2003 Aug;25(4):335-42. 
6. Miura F, Mogi M, Ohura Y, Hamanaka H. The super-elastic property of the Japanese NiTi alloy wire for use in orthodontics. Am J Orthod Dentofacial Orthop. 1986 Jul;90(1):1-10.

7. Miura F, Mogi M, Ohura Y, Karibe M. The super-elastic Japanese NiTi alloy wire for use in orthodontics. Part III. Studies on the Japanese NiTi alloy coil springs. Am J Orthod Dentofacial Orthop. 1988 Aug;94(2):89-96.

8. Owman-Moll P, Kurol J, Lundgren D. Continuous versus interrupted continuous orthodontic force related to early tooth movement and root resorption. Angle Orthod 1995;65(6):395401; discussion 401-2.

9. Ballard DJ, Jones AS, Petocz P, Darendeliler MA. Physical properties of root cementum: Part 11. Continuous vs. intermittent controlled orthodontic forces on root resorption. A microcomputed-tomography study. Am J Orthod Dentofacial Orthop. 2009 Jul;136(1):8.e1-8; discussion 8-9.

10. Weltman B, Vig KW, FieldsHW,ShankerS, Kaizar EE. Rootresorption associated with orthodontic tooth movement: a systematic review. Am J Orthod Dentofacial Orthop. 2010 Apr;137(4):462-76.

11. Brezniak N, Wasserstein A. Orthodontically induced inflammatory root resorption. Part II: the clinical aspects. Angle Orthod. 2002 Apr;72(2):180-4.

12. Ioannidou-Marathiotou I, Papadopoulos MA, Kokkas A. Orthodontic treatment and root resorption of teeth: critical analysis of mechanical factors. Hell Orthod Rev 2010:13(1-2):25-42.

13. Roberts-Harry D, Sandy J. Orthodontics. Part 11: orthodontic tooth movement. Br Dent J. 2004 Apr 10;196(7):391-4; quiz 426.

14. Artun J, Van 't Hullenaar R, Doppel D, Kuijpers-Jagtman AM. Identification of orthodontic patients at risk of severe apical root resorption. Am J Orthod Dentofacial Orthop. 2009 Apr;135(4):448-55.

15. Lund H, Gröndahl K, Gröndahl HG. Cone beam computed tomography for assessment of root length and marginal bone level during orthodontic treatment. Angle Orthod. 2010 May;80(3):466-73.
16. Dudic A, Giannopoulou C, Leuzinger M, Kiliaridis S. Detection of apical root resorption after orthodontic treatment by using panoramic radiography and cone-beam computed tomography of super-high resolution. Am J Orthod Dentofacial Orthop. 2009 Apr;135(4):434-7.

17. Heller IJ, Nanda R. Effect of metabolic alteration of periodontal fibers on orthodontic tooth movement. An experimental study. Am J Orthod. 1979 Mar;75(3):239-58.

18. Verna C, Zaffe D, Siciliani G. Histomorphometric study of bone reactions during orthodontic tooth movement in rats. Bone. 1999 Apr;24(4):371-9.

19. Miyoshi K, Igarashi K, Saeki S, Shinoda H, Mitani H. Tooth movement and changes in periodontal tissue in response to orthodontic force in rats vary depending on the time of day the force is applied. Eur J Orthod. 2001 Aug;23(4):329-38.

20. Kameyama T, Matsumoto Y, Warita H, Soma K. Inactivated periods of constant orthodontic forces related to desirable tooth movement in rats. J Orthod. 2003 Mar;30(1):31-7.

21. Hayashi H, Konoo T, Yamaguchi K. Intermittent 8-hour activation in orthodontic molar movement. Am J Orthod Dentofacial Orthop. 2004 Mar;125(3):302-9.

22. Cuoghi OA, Tondelli PM, Sonoda CK, Aiello CA, Mendonça MR, Costa SC. Induction of ankylosis in the incisor for orthodontic tooth movement in rats.Dent Traumatol. 2014 Apr;30(2):112-7. doi: 10.1111/edt.12056. Epub 2013 Jul 7.

23. Houston WJ. The analysis of errors in orthodontic measurements. Am J Orthod. 1983 May;83(5):382-90.

24. Zar JH. Biostatistical analysis. 5th ed. New Jersey: PrenticeHall; 2009. 994 p.

25. Cuoghi OA, Tondelli PM, Aiello CA, Mendonça MR, Costa SC. Importance of periodontal ligament thickness. Braz Oral Res. 2013 Jan-Feb;27(1):76-9. 